\title{
Plant polyamines in stress and development: an emerging area of research in plant sciences
}

\author{
Rubén Alcázar* and Antonio F. Tiburcio \\ Unitat de Fisiologia Vegetal, Departament de Productes Naturals, Biologia Vegetal i Edafologia, Facultat de Farmàcia, Universitat de Barcelona, Barcelona, Spain \\ *Correspondence: ralcazar@ub.edu
}

Edited and reviewed by:

Joseph M. Jez, Washington University in St. Louis, USA

Keywords: polyamines, putrescine, spermidine, spermine, thermospermine, transglutaminase, stress, ROS

Compelling evidence indicates the participation of polyamines in abiotic and biotic stress responses in plants. Indeed, genetic engineering of polyamine levels in plants has successfully improved biotic and abiotic stress resistance in model plants and crops. We anticipate that many of the current challenges in agriculture to cope with climate change and maintain nutritional quality of fruits and vegetables can be approached by considering the polyamine pathway.

The polyamine field is very dynamic as demonstrated in the large number of monthly publications in all disciplines studying polyamines (including plant sciences, human health, and microbiology). It is composed by a broad spectrum of research laboratories spread around the world, which have provided important contributions into mechanistic processes, present and future practical applications. Still, some areas remain to be explored which makes this a fascinating topic in plant sciences. In this topic, the Editors aimed at establishing a broad perspective of polyamine action in plant stress and development by inviting key researchers in the field. We would like to thank all contributors for joining us in this special topic in Frontiers in Plant Science and we hope that authors have enjoyed the interactions and discussions with editors and reviewers around their excellent works.

This topic contains five reviews, five original research studies and one hypothesis and theory article. Minocha et al. (2014) provides a review update about the complex relationship between polyamines and abiotic stress tolerance with selected examples of polyamine genetic engineering that improve tolerance traits, the concept of stress priming and interactions of polyamines with ROS and other signaling pathways. Do et al. (2014) analyze the polyamine transcriptome and metabolome in rice cultivars differing in salt tolerance, which provides an interesting comparison with potential applications in plant breeding. The interactions between biotic stress and polyamines are reviewed by Jiménez-Bremont et al. (2014) who synthesizes the current knowledge of polyamine metabolism in compatible and incompatible interactions, discusses about the capacity of phytopathogenic microbes of modulating polyamine metabolism for their own benefit, interactions with beneficial microorganisms and practical applications to induce biotic stress tolerance. Marco et al. (2014) reports that overexpression of SAMDC1 enhances the expression of defense-related genes in Arabidopsis and promotes disease resistance against bacterial and oomycete pathogens. Another complementary perspective, Valdés-Santiago and Ruiz-Herrera (2014) provide an original and illustrative view on recent advances about polyamine metabolism in fungi, ranging from mutant characterization to potential mechanisms of action in response to various stresses in selected fungal models. Although free polyamines often capture most of our attention, polyamines are present in free and bound forms resulting from interactions with cellular macromolecules. Some of these interactions occur by covalent linkages with specific proteins in reactions catalyzed by transglutaminases (TGase). Del Duca et al. (2014) provide an original review about the role of TGase on senescence and cell death in various plant models. Interestingly, the role of plant TGase is mediated by a similar molecular mechanism described for apoptosis in animal cells, which opens an interesting field for further exploration in the future. In the context of mechanistic processes, accumulating evidence suggests that polyamines play essential roles in the regulation of plant membrane transport. The review by Pottosin and Shabala (2014) summarizes the effects of polyamines and their catabolites (i.e., ROS) on cation transport across plant membranes, and discuss the implications of these effects for ion homeostasis, signal-transduction, and adaptive responses of plants to environmental stimuli. The regulation of ROS homeostasis by the polyamine back-conversion pathway catalyzed by polyamine oxidase 3 (PAO3) has been investigated by Andronis et al. (2014) in an original article. From a developmental perspective, Tong et al. (2014) provide evidence for the modulation of auxin signaling by thermospermine, which sheds light into polyamine mechanisms of action on plant development. In ripening apple fruit, Deyman et al. (2014) report the interaction of polyamines with products of polyamine catabolism (i.e., GABA). Traditionally, polyamines are described as organic polycations, when in fact they are bases that can be found in a charged or uncharged form. Although uncharged forms represent less than $0.1 \%$ of the total polyamine pool, Ioannidis and Kotzabasis (2014) propose that the physiological role of uncharged polyamines could be crucial in chemiosmosis. The authors explain the theory behind polyamine pumping and ion trapping in acidic compartments (i.e., the lumen of chloroplast) and how this regulatory process could improve either photochemical efficiency and the synthesis of ATP or fine tune antenna regulation and make plants more tolerant to stress. 


\section{ACKNOWLEDGMENTS}

Rubén Alcázar acknowledges support from the Ramón y Cajal Program (RYC-2011-07847) of the Ministerio de Ciencia e Innovación (Spain) and the Marie Curie Career Integration Grant (DISEASENVIRON, PCIG10-GA-2011-303568) of the European Union. Antonio F. Tiburcio acknowledges support by the Spanish Ministerio de Ciencia e Innovación (BIO201129683 and CSD2007-00036) and the Generalitat de Catalunya (SGR2009-1060).

\section{REFERENCES}

Andronis, E. A., Moschou, P. N., Toumi, I., and Roubelakis-Angelakis, K. A. (2014). Peroxisomal polyamine oxidase and NADPH-oxidase cross-talk for ROS homeostasis which affects respiration rate in Arabidopsis thaliana. Front. Plant Sci. 5:132. doi: 10.3389/fpls.2014.00132

Del Duca, S., Serafini-Fracassini, D., and Cai, G. (2014). Senescence and programmed cell death in plants: polyamine action mediated by transglutaminase. Front. Plant Sci. 5:120. doi: 10.3389/fpls.2014.00120

Deyman, K. L., Brikis, C. J., Bozzo, G. G., and Shelp, B. J. (2014). Impact of 1-methylcyclopropene and controlled atmosphere storage on polyamine and 4-aminobutyrate levels in "Empire" apple fruit. Front. Plant Sci. 5:144. doi: 10.3389/fpls.2014.00144

Do, P. T., Drechsel, O., Heyer, A. G., Hincha, D. K., and Zuther, E. (2014). Changes in free polyamine levels, expression of polyamine biosynthesis genes, and performance of rice cultivars under salt stress: a comparison with responses to drought. Front. Plant Sci. 5:182. doi: 10.3389/fpls.2014. 00182

Ioannidis, N. E., and Kotzabasis, K. (2014). Polyamines in chemiosmosis in vivo: a cunning mechanism for the regulation of ATP synthesis during growth and stress. Front. Plant Sci. 5:71. doi: 10.3389/fpls.2014.00071

Jiménez-Bremont, J. F., Marina, M., Guerrero-González, M. L., Rossi, F. R., Sánchez-Rangel, D., Rodríguez-Kessler, M., et al. (2014). Physiological and molecular implications of plant polyamine metabolism during biotic interactions. Front. Plant Sci. 5:95. doi: 10.3389/fpls.2014.00095

Marco, F., Busó, E., and Carrasco, P. (2014). Overexpression of SAMDC1 gene in Arabidopsis thaliana increases expression of defense-related genes as well as resistance to Pseudomonas syringae and Hyaloperonospora arabidopsidis. Front. Plant Sci. 5:115. doi: 10.3389/fpls.2014.00115

Minocha, R., Majumdar, R., and Minocha, S. C. (2014). Polyamines and abiotic stress in plants: a complex relationship. Front. Plant Sci. 5:175. doi: 10.3389/fpls.2014.00175

Pottosin, I., and Shabala, S. (2014). Polyamines control of cation transport across plant membranes: implications for ion homeostasis and abiotic stress signaling. Front. Plant Sci. 5:154. doi: 10.3389/fpls.2014.00154

Tong, W., Yoshimoto, K., Kakehi, J.-I., Motose, H., Niitsu, M., and Takahashi, T. (2014). Thermospermine modulates expression of auxin-related genes in Arabidopsis. Front. Plant Sci. 5:94. doi: 10.3389/fpls.2014.00094

Valdés-Santiago, L., and Ruiz-Herrera, J. (2014). Stress and polyamine metabolism in fungi. Front. Chem. 1:42. doi: 10.3389/fchem.2013.00042

Conflict of Interest Statement: The authors declare that the research was conducted in the absence of any commercial or financial relationships that could be construed as a potential conflict of interest.

Received: 06 June 2014; accepted: 16 June 2014; published online: 03 July 2014.

Citation: Alcázar R and Tiburcio AF (2014) Plant polyamines in stress and development: an emerging area of research in plant sciences. Front. Plant Sci. 5:319. doi: $10.3389 / \mathrm{fpls} .2014 .00319$

This article was submitted to Plant Metabolism and Chemodiversity, a section of the journal Frontiers in Plant Science.

Copyright (C) 2014 Alcázar and Tiburcio. This is an open-access article distributed under the terms of the Creative Commons Attribution License (CC BY). The use, distribution or reproduction in other forums is permitted, provided the original author(s) or licensor are credited and that the original publication in this journal is cited, in accordance with accepted academic practice. No use, distribution or reproduction is permitted which does not comply with these terms. 